\title{
Video-Based Person Re-Identification: Methods, Datasets, and Deep Learning
}

\author{
Manisha Talware, Sanjay Koli
}

\begin{abstract}
Video Analytics applications like security and surveillance face a critical problem of person re-identification abbreviated as re-ID. The last decade witnessed the emergence of large-scale datasets and deep learning methods to use these huge data volumes. Most current re-ID methods are classified into either image-based or video-based re-ID. Matching persons across multiple camera views have attracted lots of recent research attention. Feature representation and metric learning are major issues for person re-identification. The focus of re-ID work is now shifting towards developing end-to-end re-Id and tracking systems for practical use with dynamic datasets. Most previous works contributed to the significant progress of person re-identification on still images using image retrieval models. This survey considers the more informative and challenging video-based person re-ID problem, pedestrian re-ID in particular. Publicly available datasets and codes are listed as a part of this work. Current trends which include open re-identification systems, use of discriminative features and deep learning is marching towards new applications in security and surveillance, typically for tracking.
\end{abstract}

Index Terms-Person Re-Identification, Camera Network, Video Analytics, Deep Learning, pedestrian detection.

\section{INTRODUCTION}

With the spread of large networks of CCTV and surveillance cameras, it is beyond the capacities of a human operator to track an individual. Automated tracking systems are used to identify and tag a particular person by reidentifying. To identify the same human being across different frames of videos or still images is an important, but challenging task in intelligent video surveillance $[1,2]$. The main objective of re-identification system is to find a person who appeared at instances and locations in the nonoverlapping camera network.

The basics of person re-identification abbreviated as reID are to compare a sought person or group(s) as seen in query image to a dataset or gallery of persons or group(s). If a person or group(s) in the query exist in the gallery, it results in a high matching ratio or higher index of similarity or rank compared to others. This involves inter person matching and intra-person matching both. Though person Re-ID is fundamentally similar to the image retrieval task, inter camera and intra-camera variances of viewpoint, illumination, occlusion, variations in pose, makes person Re-ID a considerably challenging issue to address [1]. Videos characteristically contain additional information than still images, video-based person re-identification has proved tougher with a new set of dimensions and challenges, turn out to be appealing for researchers [2].

Revised Manuscript Received on February 25, 2020.

Manisha Talware, Research Scholar at G.H. Raisoni College of Engineering and Management, Pune, India

Dr Sanjay Koli, Professor, D. Y. Patil Inst. of Info. Technology and Research Supervisor at G.H. Raisoni College of Engineering and Management, Pune, India
Image-based person re-ids are generally categorized in feature representation and distance learning-based methods. Former aims at extracting distinctive features from pedestrian images like salience, midlevel, and salient colour features. The latter work to explore effective distance metrics, to increase matching accuracy based on the similarity index of two images. Recent methods include Large Margin Nearest Neighbor (LMNN), Relative Distance Comparison (RDC) and few others. Person re-id methods working on distance metrics learning are found effectiveness. However, these methods are basically evolved for image-based. These methods do not consider intricacies in video-based identification tasks. Person re-identification (re-ID) is one of the critical research area in video analytics, domains like security and surveillance in particular [2]. In real-life end-to-end surveillance arrangements, query image candidates are generated on-the-fly, resulting in dynamic gallery sets. As the surveillance video consists of numerous hours of videos and tons of person images, a scalable person Re-ID system with better efficiency along with generalization ability [1] is very much required to be designed. Apart from the volume, there exist large variation between different videos of pedestrian, even it is observed within each video, too. These variations make it a challenging problem to conduct re-ID between such pedestrian videos [3]. For significant improvement in the performance of Re-ID, generating robust descriptions [4], learning discriminative distance metrics [5], and powerful classifiers with good training is required. In practical, a video provides much information, so is a more natural method for person re-identification.

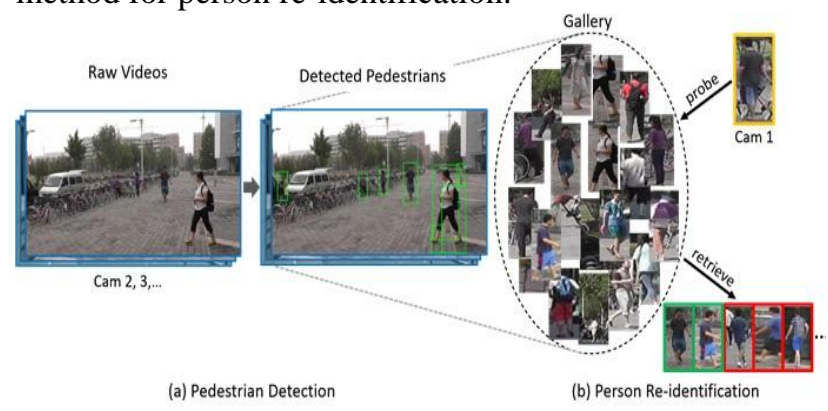

Figure 1: Person Re-ID in Videos: End-to-End [6]

Figure 1 illustrates the problem of person re-ID in videos. Severe deviations are observed between different sequences of the same individual [7] as reported in the iLIDS-VID dataset. [7] Large variations are also observed between different frames of the same video. The deviations among different videos of the same individual are termed as an inter-video variation. Variations in different frames of the same video are referred to as intra-video variations.

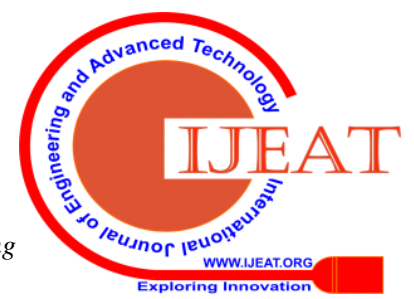


Both of these variations adversely affect matching [3]. The video contains image sequences, temporal priors in connection to a person's movements, like one's gait and pose. This information helps out to bring clarity in challenging cases for recognizing a look-alike but not different persons in a diverse camera network $[1,3,6]$. Sequences of images make more samples available for a person's appearance. Individual sample image may have different pose and viewpoint. This creates enough space to construct a more reliable appearance-based model.

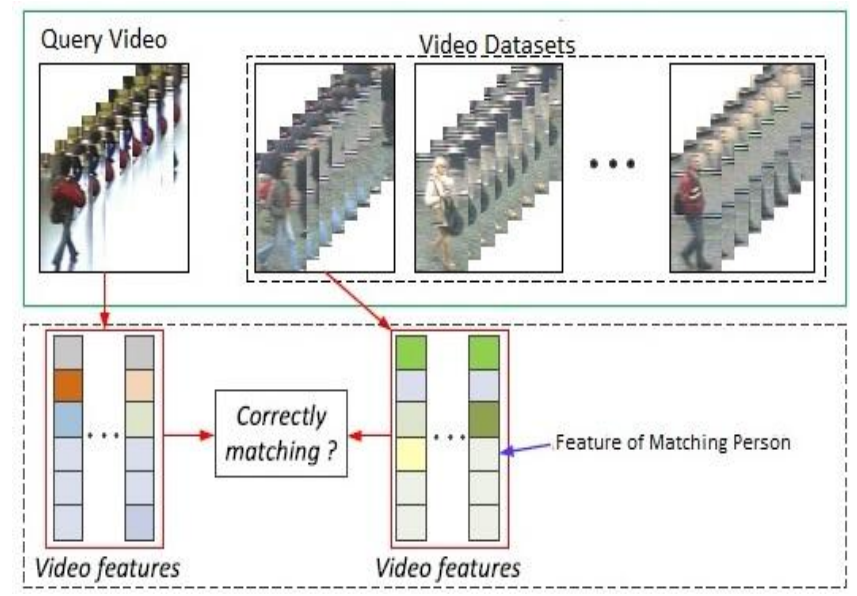

Figure 2: Illustration of the process of video-based person re-identification. [3]

Video-based person re-ID involving extraction of discussed in these approaches concentrate on Spatiotemporal feature identification and extraction, which is most representative of the pedestrian video under test. Later, video-based person re-identification is carried out with the help of these features. Figure 2 represents an example method of video-based person re-ID. It typically generates maximum possible fragments in order to extract the Spatiotemporal feature from each one if it. These smaller sequences characterize pedestrian video by virtue of Spatiotemporal features extracted from the fragments $[9,10]$. This takes a form of set matching problem for re-ID of the pedestrian videos.

\section{RELATED WORKS}

The journey of Person re-Identification research fueled with a multi-camera object tracking, way back in 1997. It is where the term "person re-identification" is coined, was on record in 2005. Ghesissari et al. designed a Spatio-temporal segmentation using the video Frames, but Matching was based on images [11]. Video-based re-ID methods put more emphasis on multi-shot matching. These methods integrate information extracted from the temporal domain with a spatial one, making it a case of Spatio-temporal matching. Later Li. et al proposed the use of siamese neural networks with limited training samples for determining whether different probed images belong to the same person ID [12].

\section{A. Available Datasets}

In order to systematize the process and evolve benchmarks, certain datasets are made available publicly by researchers and Institutes in the field. Most of them are even annotated. Datasets used for academic purpose are consists Spatio-temporal features are discussed in [7, 9, 10]. Methods

of images taken from cameras with optical axes parallel to the ground plane, whereas, in real-world scenes, the angle of capture is much larger because of constraints on camera installations. [2]. Few of the publicly available datasets for person re-identification include VIPeR [8], GRID [13], CAVIAR [14], DukeMTMC4ReID [15], 3DPeS [16], Market1501 [17], PRID [18], V47 [19], WARD [20], SAIVT-SoftBio [21], CUHK01, CHUK02, CHUK03 [22], RAiD [23], iLIDS VID [7], HDA+ [24], Airport [2] , SYSU-sReID [25].

Out of these datasets, most are built for academic purposes, whereas very few datasets of real-world surveillance are available publicly. Most of these datasets are manually annotated $[8,13,17,18]$ to contain sets of bounding boxes for the queries and conforming matching candidates. Whereas, real-world end-to-end surveillance network incorporates automatic detection as well as tracking units $[2,3]$.

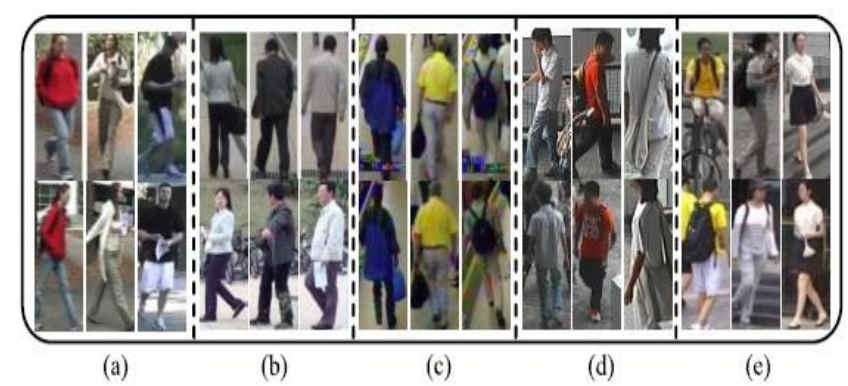

Figure 3: Sample Images from a)VIPeR b)SYSU-sReID c) GRID d)CHUK03 and e)Market1501. Row 1 and two are for the same person from a different view.

Dataset annotations usually involve challenges like variations in viewpoint, variation in illumination, errors in detection, occlusion, background clutter, etc. Srikrishna et al described the characteristics of most of these publicly available datasets in a tabulated manner. This paper also presents a good comparison of these datasets [2].

While working on datasets with large volume and challenges of variations, most of the methods concatenate on improving the Re-ID accuracy but pay little attention to the algorithm efficiency and its ability to generalize. This is equally important in practical systems. [1].

Table 1 lists a few of publicly available databases with distinct and characterizing information about these galleries. Annotation methods are either hand-curated or Aggregated Channel Features (ACF) [2]. Variations are Viewpoint Variation (VV) [35], Illumination Variation abbreviated as IV, OCC for Occlusion, BC for Background Clutter, RES is for variation in image resolution are few short names used in the table. Single or Multi indicates no. of shots [2, 3, 27].

\section{B. Re-Identification Methods:}

Methods widely used for person re-identification include feature extraction based, distance metric learning-based and one involves the use of Deep learning networks, Convolutionary neural networks in particular. These methods are graphically represented in figure 4.

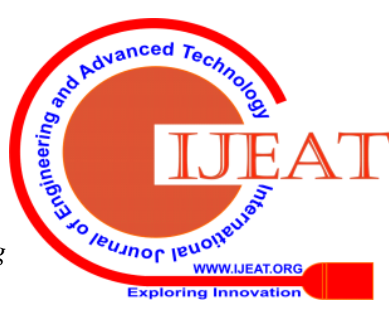




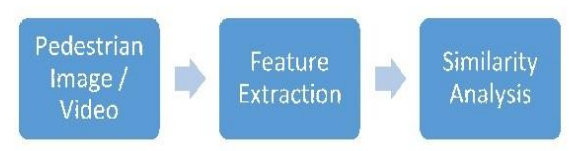

Figure 4a: Feature based methods of re-ID

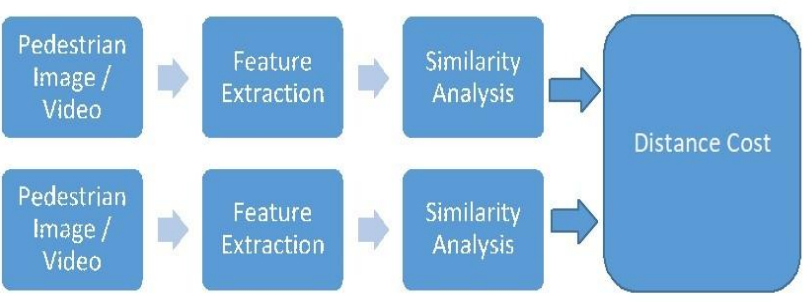

Figure 4b: Metric based methods of re-ID

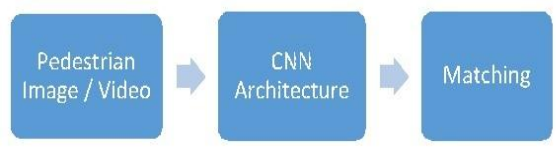

Figure 4c: CNN based methods of re-ID

\section{Figure 4: Different methods of person re-identification}

\section{Learning-based Approaches:}

Re-ID system has basically two parts i.e. robust feature identification, extracting it and another is metric learning. It pulls the samples of the same class nearer while pushing the samples of different classes distant from each other. CNN has been used in many people Re-ID works like [5]. Person Re-ID has been seen as a classical image retrieval problem. The key component in the framework of large scale person re-identification proposed by [1] is the Convo-Net, which extracts discriminative deep features. Focus is on multicamera, multi-shot person Re-ID using large-scale image retrieval. The author proposes a Coarse-to-Fine (C-F) framework for Re-ID. Convo-Net generates a dense descriptor for the coarse-level search. It also generates an equally robust descriptor for searching at fine-level.

A robust and efficient approach id developed by Lisanti et al. for Re-ID. The method is built on a sparse basis. Though data is high dimensional, images/sequences from the same class are observed to converge on the same lowdimensional subspace, which belongs to original feature space [26]. A descriptor of person appearance, exhibit its strength against pose and illumination variations. The sparse discriminative framework and robust descriptor improves the rank performance and is capable of single-shot Re-ID. The iterative sparse ranking finds its application in many other fields along with person Re-ID. A proposal to learn simultaneous intra-video and inter video distance learning (SI2DL) is recommended by [3]. This technique learns metric of an intra and inter-video distance. All distance learning metrics aim at separating the videos, which are of different persons, but truly matching. Intra-video distance makes each video more compact. Inter video distance is to take truly matching videos closer. A pair of separation-based distance learning like P-SI2DL is further proposed by [3]. Comparison of video-based and set-based methods with experiments on iLIDS-VID [7], PRID2011 [18], MARS [27] and HDA+ [24] is discussed and the superiority of the SI2DL approach is commented.

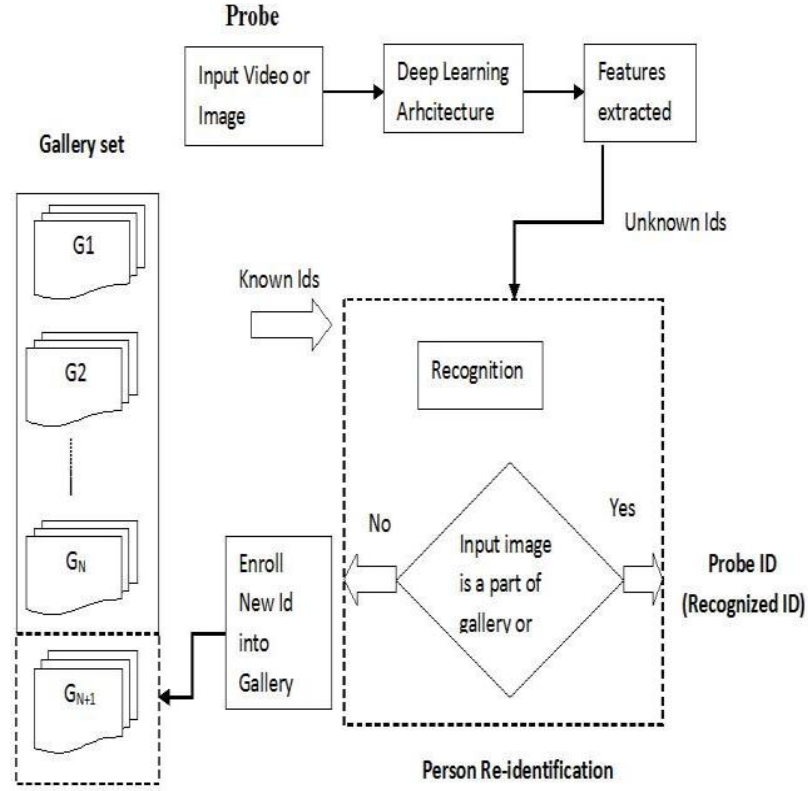

Figure 5: Process of Person Re-identification using deep learning approach [28]

Figure 5 explains the general arrangement in different approaches used in deep learning techniques for person reID. This involves a gallery set with multiple videos stored for different persons or groups at different times. A probe is mostly used to extract features and pass these to recognition system, which either recognizes if similarity score is good with one or more images/videos or it asks to add a new identity of a pedestrian for future detection. A survey is presented by Muna et al in [29].

In a Multi-Rate Gated RCNN for Video-Based Pedestrian Re-Identification approach proposed by Zhihui et al. [30], a video of a pedestrian re-identification in nonoverlapping cameras. The method employs a CNN to extract the features from appearance and motion in raw video sequences, and then feed them into a multi-rate RCNN to exploit the temporal correlations. Optical flow, colour features are used together with a recurrent layer. It proposes context reconstruction to largely improve the performance. However, the algorithm does not consider problems due to background clutter and occlusion, camera view, changes and lighting conditions.

Most of the supervised learning algorithms are domainspecific. They may give a poor performance for other domain data. Universal Model Learning (UML) for domain-generic universal person re-ID in a "train once, run everywhere" pattern is presented by [31]. It uses re-ID using only limited training data of a "single" seed domain. This method, therefore, opens up a direction taking intelligent learning algorithms closer to a real scene. Performance achieved is inferior to that of supervised learning but is more generalized [31] 
Video-Based Person Re-Identification: Methods, Datasets, and Deep Learning

Table 1: Comparison of Datasets used in person re-ID

\begin{tabular}{|c|c|c|c|c|c|c|c|c|}
\hline Dataset & $\begin{array}{l}\text { Image / } \\
\text { Video }\end{array}$ & $\begin{array}{l}\text { No. of } \\
\text { Persons }\end{array}$ & $\begin{array}{l}\text { Annotation } \\
\text { Method }\end{array}$ & $\begin{array}{l}\text { No. of } \\
\text { Camera }\end{array}$ & $\begin{array}{l}\text { No. of } \\
\text { Images }\end{array}$ & Variations & $\begin{array}{l}\text { No. of } \\
\text { Shots }\end{array}$ & Link, if publicly available \\
\hline VIPeR & Images & 632 & Hand & 02 & 1264 & VV, IV & Single & https://vision.soe.ucsc.edu /node/ 178 \\
\hline GRID & & 1025 & Hand & 08 & 1275 & $\begin{array}{l}\text { VV,BC,OC } \\
\text { C,RES }\end{array}$ & Multi & $\begin{array}{l}\text { http://personal.ie.cuhk.edu.hk/ ccloy/ } \\
\text { downloads qmul underground reid.html }\end{array}$ \\
\hline PRID11 & $\begin{array}{l}\text { Image / } \\
\text { Video }\end{array}$ & & Hand & & & VV, IV & Multi & $\begin{array}{l}\text { https://www.tugraz.at/institute/icg/researc } \\
\text { h/team-bischof/lrs/downloads/prid11 }\end{array}$ \\
\hline iLIDs-VID & $\begin{array}{l}\text { Image / } \\
\text { Video }\end{array}$ & 300 & Hand & 02 & 42495 & $\begin{array}{l}\text { VV,IV,BC, } \\
\text { OCC }\end{array}$ & Multi & $\begin{array}{l}\text { http://www.eecs.qmul.ac.uk/ xiatian } \\
\text { /downloads_qmul_iLIDS- } \\
\text { VID_ReID_dataset.html }\end{array}$ \\
\hline $\begin{array}{l}\text { HDA } \\
\text { Person }\end{array}$ & $\begin{array}{l}\text { Image / } \\
\text { Video }\end{array}$ & 53 & $\begin{array}{l}\text { Hand /Pyramid } \\
\text { features }\end{array}$ & 13 & 1976 & VV,IV,DE & & http://vislab.isr.ist.utl.pt/ datasets/\#hda \\
\hline Airport & $\begin{array}{l}\text { Image / } \\
\text { Video }\end{array}$ & 9651 & $\mathrm{ACF}$ & 06 & 39902 & $\begin{array}{l}\text { VV,IV,DE, } \\
\text { BC,OCC }\end{array}$ & $\begin{array}{l}\text { Multi } \\
\text { Resolution } \\
128 * 64\end{array}$ & $\begin{array}{l}\text { http://www.northeastern.edu/alert/ } \\
\text { transitioning-technology/alert-datasets/ } \\
\text { alert-airport-re-identification-dataset/ } \\
\text { (Available on request) }\end{array}$ \\
\hline $\begin{array}{l}\text { SAIVT- } \\
\text { SoftBio }\end{array}$ & Video & 152 & Hand & 08 & 64472 & $\mathrm{VV}, \mathrm{IV}, \mathrm{BC}$ & Multi & $\begin{array}{l}\text { https://data.researchdatafinder.qut.edu.au/ } \\
\text { dataset/saivt-soft-biometric }\end{array}$ \\
\hline $\begin{array}{l}\text { Market150 } \\
1\end{array}$ & $\begin{array}{l}\text { Image / } \\
\text { Video }\end{array}$ & 1501 & Hand & 06 & 32217 & VV,DE,RES & Multi & $\begin{array}{l}\text { http://www.liangzheng.com.cn/Project/pro } \\
\text { ject_reid.html }\end{array}$ \\
\hline MARS & Video & 1261 & Hand & 06 & 1191003 & $\begin{array}{l}\mathrm{DPM} \\
\text { GMMCP }\end{array}$ & $\begin{array}{l}\text { Mult } \quad- \\
\text { Reso } \\
256^{*} 128\end{array}$ & $\begin{array}{l}\text { http://www.liangzheng.com.cn/Project/pro } \\
\text { ject_mars.html }\end{array}$ \\
\hline WARD & Image & 70 & Hand & 03 & 4786 & IV & $\begin{array}{l}\text { Multi } \\
128 * 64 \\
\end{array}$ & $\begin{array}{l}\text { https://github.com/iN1k1/CVPR2012/tree/ } \\
\text { master/toolbox/Datasets/WARD }\end{array}$ \\
\hline MSMT17 & & 4101 & & & 126441 & RCNN & $\begin{array}{l}\text { Multi } \\
\text { resolution }\end{array}$ & $\begin{array}{l}\text { http://robustsystems.coe.neu.edu/sites/robu } \\
\text { stsystems.coe.neu.edu/files/systems/projec } \\
\text { tpages/reiddataset.html }\end{array}$ \\
\hline
\end{tabular}

\section{Representation Based Methods}

Re-ID model-based introduced by Lei et al in [32] uses Semantic Region Representation (SRR). Model focus on identifying a specific person in Multi-camera setup. SRR parse the person body in multiple sub-parts. This is to focus on the foreground image and pay less attention to interferences from the background [32].

This helps effective comparison of similarity between the corresponding sub-parts of the human body [32]. Mapping Space Topology Constraint (MSTC) is devised as an effective metric learning. This is to measures the similarity among images with viewpoint variations. This differentiates the intra-class variations over the inter-class variations. This work can be extended for video-based ReID [35]. Video sequence utilizes the Spatio-temporal signal for similarity indexing of pair sequences of individuals. MSTC constraint can be further extended to varied applications with deep-learning of features.

To report the similarity or dissimilarity in clear terms is one challenging issue, several researchers tried addressing this issue with the help of Spatio-temporal features representing these videos [33]. The method proposed in an article by Wei et al. uses the notion of "mean-body". In order to quantify variations between spatial and temporal features of the same video, a term of an intra-video loss is coined. These along with Siamese loss is combined to train the deep learning model right from scratch. Proposed Deep learning architecture jointly exploits intra and inter-video distances. The iterative process updates mean-body up to maximisation of accuracy on validation sets. Method claims higher accuracy on algorithms in PRID2011 [18], iLIDSVID [7] and MARS [27], WARD [36], MSMT17[37] datasets.

A model trained on one family of datasets is observed lacking in its ability to generalize on another dataset.
Overtraining loses generalization on variations between features, even for the same video.

Sometimes, re-identification needs to find the match of a suspected person image from long-running videos. A single image of such a person is usually provided to reidentify from a huge number of pedestrian videos. This is usually referred to as an image to video person Re-ID (IVPR). Zhu et al. proposed [34] a method for it by learning heterogeneous dictionary pairs along with a matrix of feature projection. It uses a joint feature projection matrix for matching heterogeneous features from image and video. Attempt to reduce the effect of intra-video variations on the re-ID. Image-video triplet (IVT) defines the relationship in image and video. This approach incorporates additional computational burden and training time.

\section{DISCUSSION}

Re-Identification can be perceived as a problem of Maximization: Let us consider model, Where G represents a gallery (database) consists of $\mathrm{N}$ images identified as $\left\{g_{i}\right\}_{i=1}^{N}$, with query image denoted as q. Image identification / uniqueness is calculated by:

$i^{*}=\operatorname{argmax}_{i \in 1,2, \ldots N} \operatorname{sim}\left(q, g_{i}\right) \quad------E q .01$

Where $i$ is the representation of probe q. Sim(_; _ ) denotes similarity function. This represents image description and distance metric for image-based person re-ID. In videobased re-ID sets of bounding boxes replaces images $\mathrm{q}$ and $\mathrm{g}$.
$\left\{q_{i}\right\}_{i=1}^{N q}$
$\left\{g_{j}\right\}_{j=1}^{N g}$

Where $\mathrm{n}_{\mathrm{q}}$ and $\mathrm{n}_{\mathrm{g}}$ are representing bounding boxes within a video sequence. 
In the real-world scenes, the end-to-end re-ID system uses un-processed videos as input, incorporates pedestrian detection and tracking algorithms, along with models for reID. Initial re-ID works presume that the datasets of pedestrian bounding boxes is hand-annotated with perfect detection and is always available.

Pedestrian detectors are inherently contain few errors including one with the bounding boxes such as misalignment, missing detection of correct individual, and false-positive alarms. Outlier frames within a tracklet are due to tracking errors within trackers.

Person identification i.e. re-ID accuracy on multiple openly available representative datasets, during last decade for both manually annotated and ACF, deep features are presented in survey report presented by Liang et al in 2016. Performance improvement is observed over the years and is graphically presented in [6]. Deep learning techniques [28] outperform hand-annotated systems and yield a new state of the art in most of the cases and in particular for large volume datasets. Still, there is scope for performance improvement on larger datasets, as better Rank-1 accuracy is observed to be good, it is difficult to find hard positives and to achieve a high recall. Deep learning has got inherent limitations in re-ID due to a lack of training data. Many of re-ID datasets like VIPeR [8] usually provide a pair of image for each person, this leads to the adoption of the Siamese model for re-ID

To investigate the generalization capability of any feature-based method [32] or metric learning method or Deep Convolutional Neural Networks or Recurrent Neural Networks or its variants, it is very important to have cross dataset evaluation. There exist a large variation in the data capture process and even data distribution of different datasets. So it is quite difficult to transfer the metric trained in the source (training dataset) domain to the target (validation or test dataset) domain. If tried so, one cannot expect satisfactory results due to variances of second-order, which is still an open research problem.

\section{RESULTS}

The paper discusses different methods used for reidentification. It basically starts with categorizing problems of person re-id and identifying it as a research area. Though it comes under computer vision or pattern recognition and tracking, it has created its own space in image and video analytics. The timeline starts two decades back and now moved towards end-to-end video surveillance systems with the deployment of research work. It is extending wing towards crowd identification and 'Internetification' which incorporates images from various sources like social media profile photos. While moving from closed to open world reID, limitations of benchmark datasets are getting surfaced. Spatiotemporal relationship of camera and exploitation of environmental knowledge is gaining importance. Model learning has to be generalized with optimal metrics. Deep learning of features and variations is coming up to address the issue up to a certain extent.

\section{Conclusion}

The current trends and issues with person re-ID can be summarized as:

1. Evaluation of system performance: Designing evaluation metrics for pedestrian detection and tracking is still a critical issue in re-ID. Intersect over Union (IoU) score is important criteria, Still, consensus to be formed on perfect single frame detector and the automatic detection has to be resolved. Evaluation of tracking under person re-ID is an open research area.

2. The gallery of images /videos itself is getting dynamic with the advent in video capture tools and camera technology. Gallery size impacts the detection accuracy by and large. Though rank matching and detection recall are few metrics of evaluation, there are new evaluation metrics that are coming up and these shall be informative and unbiased.

3. To minimize the influence of Tracker/detector on re-ID: Some intrinsic mechanism and feasible solutions are required to take care for missing parts of bounding box, to deal with misalignment and global variances in observations, adaption to scale changes, missing parts from the annotated image/frame and to take care of false positives and missed out. Detections

Research of Person re-identification is currently seen standing between image classification and retrieval. Re-ID has to be more accurate and efficient in the future. Current research is in end-to-end re-ID. These re-identification integrate pedestrian detection and tracking, very large datasets with multi-shot and multiple cameras, leading toward practical systems for use.

Data volume due to continuous monitoring create problems of abundance. Re-ID re-ranking methods, open reidentification systems are a new set of challenges. The integration of deep learning with the discriminative feature, optimization of video trackers or detectors, and efficient data management may be the direction towards a successful person re-identification system.

\section{ACKNOWLEDGEMENT}

Author thanks the researchers who made the datasets and codes available publicly, to the fellow researchers. Previous reviewers of the domain area and contributors in data and methods to address the issues. All links mentioned in this paper are as made available by the respective authors/institutes.

\section{REFERENCES}

1. Hantao Yao, Shiliang Zhang et al, "Large-Scale Person ReIdentification as Retrieval" Proceedings of the IEEE International Conference on Multimedia and Expo (ICME) 2017

2. Srikrisna Karanam et al "A Systematic Evaluation and Benchmark for Person Re-Identification: Features, Metrics, and Datasets”, CoRR, abs / 1605.09653, 2016

3. Xiaoke Zhu, Xiao-Yuan Jing, Xinge You "Video-Based Person ReIdentification by Simultaneously Learning Intra-Video and InterVideo Distance Metrics", IEEE transaction on image processing, Vol 27, No. 11, Nov.2018

4. H. Liu, J. Feng, M. Qi, J. Jiang, and S. Yan, "End-toend comparative attention 


\section{Video-Based Person Re-Identification: Methods, Datasets, and Deep Learning}

networks for person re-identification," TIP, 2016.

5. Z. Zheng, L. Zheng, and Y. Yang, "A discriminatively learned cnn embedding for person re-identification," arXiv preprint arXiv:1611.05666, 2016

6. Liang Zheng, Yi Yang, Alexander G. " Person Re-identification: Past, Present, and Future" arXiv:1610.02984v1 Oct 2016

7. T. Wang et al., "Person re-id by video ranking," in ECCV, 2014

8. D. Gray, H. Tao, "Viewpoint invariant pedestrian recognition with an ensemble of localized features," in ECCV, 2008.

9. T. Wang, S. Gong, X. Zhu, and S. Wang, "Person re-identification by discriminative selection in video ranking," IEEE Trans. Pattern Anal. Mach. Intell., vol. 38, no. 12, pp. 2501-2514, Dec. 2016.

10. K. Liu, B. Ma, W. Zhang, and R. Huang, "A Spatio-temporal appearance representation for video-based pedestrian reidentification," in Proc. IEEE Conf. ICCV, Dec. 2015, pp. 38103818.

11. N. Gheissari, T. B. Sebastian, and R. Hartley, "Person reidentification using spatiotemporal appearance," in 2006 IEEE Computer Society Conference on Computer Vision and Pattern Recognition (CVPR'06), vol. 2. IEEE, 2006, pp. 1528-1535.

12. W. Li, R. Zhao, T. Xiao, and X. Wang, "Deepreid: Deep filter pairing neural network for person re-identification," in Proceedings of the IEEE Conference on Computer Vision and Pattern Recognition, 2014, pp. $152-159$

13. C. C. Loy, T. Xiang, and S. Gong, "Time-delayed correlation analysis for multi-camera activity understanding," Intl. Journal of Computer Vision, vol. 90, no. 1, pp. 106-129, 2010.

14. D. S. Cheng et al., "Custom pictorial structures for re-identification." in British Machine Vision Conference, 2011

15. M. Gou et al., "DukeMTMC4ReID: A large-scale multi-camera person re-identification dataset," in Computer Vision and Pattern Recognition Workshops, 2017.

16. D. Baltieri, R. Vezzani, and R. Cucchiara, "3DPeS: $3 \mathrm{~d}$ people dataset for surveillance and forensics," in Proceedings of the 2011joint ACM workshop on Human gesture and behaviour understanding, 2011.

17. L. Zheng et al., "Scalable person re-identification: A benchmark," in ICCV, 2015.

18. M. Hirzer et al., "Person re-identification by descriptive and discrimina classification," in Image Analysis, 2011.

19. S. Wang et al., "Re-identification of pedestrians with variable occlusion and scale," in ICCV Workshops, 2011.

20. N. Martinel, C. Micheloni, and C. Piciarelli, "Distributed signature fusion for person re-identification," in ICDSC, 2012.

21. A. Bialkowski et al., "A database for person re-identification in multicamera surveillance networks," in DICTA, 2012.

W. $\mathrm{Li}, \mathrm{R}$ Z Zhao, and X Wang "Human re-identification with transf metric learning." Springer, 2012, pp. 31-44.

23. A. Das, A. Chakraborty, and A. K. Roy-Chowdhury, "Consistent reidentification in a camera network," in ECCV, 2014.

24. D. Figueira et al., "The HDA+ data set for research on fully automated re-identification systems," in ECCV Workshops, 2014.

25. C. Guo, S.-Z. Chen, J.-H. Lai, X.-J. Hu, and S.-C. Shi, "Multishot person reidentification with automatic ambiguity inference and removal," in Proc. IEEE Int. Conf. Pattern Recognition., Aug. 2016, pp. 3540-3545.

26. G. Lisanti, I. Masi, A. D. Bagdanov and A. Del Bimbo, "Person Reidentification by Iterative Re-weighted Sparse Ranking", IEEE Transactions on Pattern Analysis and Machine Intelligence, 2015.

27. L. Zheng et al., "MARS: A video benchmark for large-scale person re identification," in Proc. ECCV, 2016, pp. 868-884.

28. Kejun Wang, Haolin Wang, Meichen Liu, Xianglei Xing, Tian Han "Survey on person re-identification based on deep learning" CAAI Trans. Intell. Technol., 2018, Vol. 3, Iss. 4, pp. 219-227

29. Muna O. AlMasawa, Lamiaa A. Elrefaei, Kawthar Moria, "A Survey on Deep Learning-Based Person Re-Identification Systems”, IEEE 10.1109/ACCESS.2019.2957336

30. Zhihui Li, Lina Yao, Feiping Nie, Dingwen Zhang, Min Xu "MultiRate Gated Recurrent Convolutional Networks for Video-Based Pedestrian Re-Identification" The 32nd AAAI Conf on AI 2018.
31. Xu Lan, Xiatian Zhu, Shaogang Gong "Universal Person Reidentification" Cs.CV C 22 July 2019.

32. Jianjun Lei, Lijie Niu, Huazhu Fu, Bo Peng "Person Re-Identification by Semantic Region Representation and Topology Constraint', IEEE Trans On C\&S For Video Technology, Vol. 29, No. 8, Aug 2019.

33. Wei Zhang, Yimeng Li, Weizhi Lu, Xinshun Xu, Zhaowei Liu, and Xiangyang $\mathrm{Ji}$ "Learning Intra-Video Difference for Person ReIdentification" IEEE Transactions On Circuits And Systems For Video Technology, Vol. 29, No. 10, October 2019.

34. Xiaoke Zhu, Xiao-Yuan Jing, Xinge You, Wangmeng Zuo, Shiguang Shan, and Wei-Shi Zheng "Image to Video Person Re-Identification by Learning Heterogeneous Dictionary Pair With Feature Projection Matrix" IEEE Transactions On Information Forensics And Security, Vol. 13, No. 3, March 2018

35. Z. Wu, Y. Li, and R. J. Radke, "Viewpoint invariant human reidentification in camera networks using pose priors and subject discriminative features," IEEE Trans. Pattern Anal. Mach. Intell., vol 37, no. 5, pp. 1095-1108, May 2014

36. N. Martinel, C. Micheloni, and C. Piciarelli, "Distributed signature fusion for person re-identification," in ICDSC, 2012.

37. Wei, L., Zhang, S., Gao, W., \& Tian, Q. (2018). Person Transfer GAN to Bridge Domain Gap for Person Re-Identification. CVPR 2018

\section{AUTHORS PROFILE}

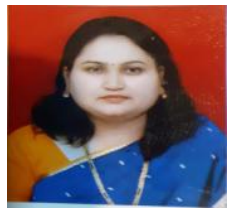

Manisha Talware, received her Bachelors Degree in Electronics and Telecommunication Engineering from North Maharashtra University, Maharashtra, India in 1999 and a Master Degree in Electronics and Telecommunication Engineering in 2011 from University of Pune, Maharashtra, India. She is currently working toward Doctoral studies at G. H

Raisoni College of Engineering and Management under S.P. Pune University, India. Her research interests include pattern recognition and Video Signal Processing, specifically the person re-identification.

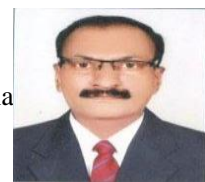

Dr S. M. Koli, has received his B.E. degree from the Department of Electronics Engineering from Shivaji University, Kolhapur, Maharashtra, India in 1997, M.

Tech. degree from the Department of Electronics and Telecommunication Engineering from Dr Babasaheb Ambedkar Technological University, Lonere, Maharashtra, India in 2006 and a Ph.D. degree in the Department of Engineering and Technology, from Sant Gadage Baba Amravati University, Amravati, Maharashtra, India in 2015. He is currently working as Professor in E\&TC at the Department of Electronics and Telecommunication Engineering at Dr D. Y. Patil School of Engineering, Pune, India and research supervisor at G. H. Raisoni College of Punedineering and Management, Pune, India. His research interests are mainly in the wireless domain and video signal processing. 